\title{
Design of a Visual Comfort Sensor for Daylighting Devices
}

\author{
M. Leclercq*, C. Anthierens**, E. Bideaux*, L Flambard*** \\ * Laboratoire AMPERE UMR 5005, Institut National des Sciences Appliquées, Lyon, France \\ (m.leclercq@sherpa-eng.com; eric.bideaux@insa-lyon.fr) \\ **Institut des Sciences du Mouvement UMR 6233, Luminy, Marseille, France \\ (cedric.anthierens@supmeca.fr) \\ *** Sherpa Engineering, La Valette du Var, France \\ (l.flambard@sherpa-eng.com).
}

\begin{abstract}
This paper reports one task of a global investigation based on a whole daylighting system designed to equip refurbished and new buildings. This study forms part of the research conducted by a French group that works on green energy. The authors aim to provide occupants with daylight for everyday tasks, hence reducing electricity consumption and increasing comfort level as well. This paper presents the conception and design of a new virtual sensor based on a fisheye video camera dedicated to the assessment of optic comfort. This device includes a fuzzy logic kernel to deal with the subjective aspect of the human perception related to sight.
\end{abstract}

\section{INTRODUCTION}

Many companies financially encouraged by their government to meet the Kyoto treaty and to decrease their energy bill, have decided to invest in new premises or refurbishments (Barlow 2007) to achieve a long-term solution. In the tertiary companies lighting and heating represent the majority of the energy consumption, which is why many companies in this field are now tempted to rely more on natural solar energy as this can supply the light and heat required. Not including the benefits of solar heating in terms of energy savings, the use of daylight can significantly help to decrease energy consumption and is also interesting thanks to its light quality.

The spectrum of sun light is very wide and provides a high power density over the whole range of visible frequencies. Therefore daylight allows us to see the true colours of our environment. Moreover when the sky is clear, the heat from sun light is high and can generate a natural comfort of sight. Humans are sensitive to surrounding light conditions and when comparing identical illumination conditions very much prefer daylight sources to artificial lights. Several criteria such as the amount of light, location of the source, heat of light etc can even affect workers' productivity and stress levels (Vischer 2005). Despite all the advantages of daylight (energy savings or light comfort), this free source of light can also cause trouble in all buildings that do not have the equipment to deal with it.

The main drawback of daylight compared to artificial light is its perpetual variation. It varies with the seasons (altitude of sun and daylight duration), time (trajectory of sun between sunrise and sunset), weather (overcast or clear sky) and environment (reflection on water or snow, presence of a building...). Several of these criteria cannot be predicted with good reliability. However, architects try to design new buildings by taking into account their orientation, the occurrence of shadows and some other basic criteria related to the local climate (Serra 1998).

Section 2 of this paper will deal with visual comfort and discomfort criteria such as glare and backlighting. Our contribution on a fuzzy optic comfort sensor is presented in the third section, from sensor conception and design to implementation. Section 4 will locate and define the role of our sensor within a control loop, which would manage the visual comfort in the experimental room. The last section will draw conclusions from the current results and introduce the forthcoming advances for the control of the whole experimental room.

\section{OPTIC COMFORT}

Many investigations have been conducted to model what we could call human optic comfort, which involves taking into account individual subjectivity to assess the comfort level. Because our behaviour tends to follow a daily biological rhythm, our comfort perception is supposed to evolve during the day. As it is described in work standards, the optic comfort level depends on the task being performed. This is why in a company the lighting in a corridor (walking hall) should be very different to the lighting of a workbench where a worker is concentrated on a microscopic task. In order to meet optic comfort criteria, we shall take into account the environment (material and people) and the task at hand in order to define the notion of optic atmosphere.

Optic comfort does not depend only on the level of light because, as we have seen above, the light temperature has an influence on the way we visually perceive our environment. Instead of describing and controlling all the criteria that lead to optic comfort according to a desired atmosphere, we can note some criteria of discomfort that it is necessary to avoid in order to tend towards a good level of optic comfort in any case and thus for any task. Glare, backlighting, shadows, 
heterogeneous lighting and too low a level of light are some criteria that contribute to increase the perception of discomfort. We detail these criteria below and present the way to deal with them in the implementation of an optical sensor to evaluate the comfort level of a room from a fixed point of view.

\subsection{Discomfort factors}

Most of the investigations conducted on glare in buildings were carried out in order to model the phenomenon, to analyse human perception or to build new premises, which can avoid the appearance of disturbing glare. The CIE has been contributing to these studies for years. Research on the understanding of human perception of glare has produced numerous models based on medical and statistical studies. The analysis of human sight should take into account the whole chain of perception from signal acquisition through the sensor (eye) to its interpretation (brain). However, it is very difficult to understand and to model this whole chain, which is why models are based on different assumptions and sometimes are not in complete agreement. Models such as CGI (Nazzal 2005) and UGR (Wienold 2006) focus on the ratio between the source luminance and the background luminance, but the technical specifications of the source (number of sources, type, dimensions, position in the field of view...) are accounted for the same way in all the models (Winold 2006). In addition to these common criteria, most models are expressed as a logarithmic function to highlight the fact that human perception is not a linear phenomenon. Experimental studies generally show that the eye reacts to glare as can be expected given the distribution of rods and cones within the retina. The density of sensitive elements is higher close to the fovea.

Even if the function of the eye as a sensor can be almost totally understood, we cannot forget that human perception is subjective and related to the individual's behaviour and his environment. Based on statistical studies, several models are written as a formal mathematical formula that does not consider the uncertainties and inaccuracies of parameters (Wonwoo 2005). However, some investigations have highlighted the influence of other parameters that are related to human subjectivity. This is the case in a study that has shown that daylight through a window cannot be accurately recreated by using an artificial source. The temperature of an artificial light cannot really simulate that of daylight. Also, an investigation has demonstrated that light with particular luminance characteristics can be tolerated by the subject when natural but becomes uncomfortable when simulated artificially (Werner 2005). Moreover, the authors of this investigation concluded that the more agreeable to look at the source is, the more bearable it is. This underlines the influence of the semantics of the watched scene. But we are interested in the subject's behaviour and environment to describe the discomfort involved by glare undergone by the subject. In this case, the type of atmosphere must be taken into account and also the task that is being undertaken. From a control point of view, we will speak about functional lighting to distinguish the light required for reading a document, chatting without backlighting, watching a screen and so on. This will highly influence the mean illumination level in the room and thus the energy consumption.

Finally human subjectivity in the perception of optic comfort depends on the ethnic origins of subjects. Indeed it has been shown that glare models statistically calibrated using experiments conducted on Caucasians cannot replicate experimental data from studies with Asians (Jin Sook 2007). According to this study, Asian people can bear more glare than Caucasian people. This illustrates the interest in calibrating the method and device i.e. the model, to assess the discomfort level in all types of case.

\section{SENSOR DESIGNING}

We have seen above that many investigations have aimed to model the discomfort mainly created by glare. The formulas generated are therefore based on current parameters such as luminance of the source / background, the size and position of the source in the field of view and also on evaluated exponents to draw a curve which matches experimental points. Based on a wealth of literature, we decided to create a sensor that would enable a continuous assessment of the level of optic discomfort in a room. This type of sensor has to provide an objective and consistent measure that would supply a control loop with a feedback or would directly give an assessment to characterise a room for example. To build most of the models quoted above, a high number of sensors such as luminancemeters and luxmeters were required. Realistically it is not possible to use so much equipment for online control of the optic atmosphere within a room as this would be a very expensive solution.

Our sensor, composed of a fisheye video camera, provides wide pictures to simulate the human field of view and it can be moved in the room to enable measurement of any point of view within the concerned room. This is very helpful to compare the points of view of two people in a face to face meeting for instance. The sensor will not take the same types of measurements as specific optic sensors but thanks to models already established, it provides an image of the comfort level or the discomfort level. Indeed even from a control point of view, the authors decided to work with the discomfort criteria in order to move towards more comfortable atmospheres by eliminating uncomfortable configurations. Thus the visual sensor is designed to detect discomfort criteria and assess the discomfort level of the scene as seen from a specific point of view.

\subsection{Sensor description}

In order to design our sensor, we have taken advantage of models of glare like CGI or UGR but also of our description of a comfortable atmosphere. Our main goal is to control the optic comfort in a room like an open office within a tertiary company, which is why we focus only on a work atmosphere where workers are supposed to perform different tasks such as reading, writing on paper, working on a computer and chatting. We will consider the chatting and reading tasks because the conditions required to properly perform those tasks depend directly on the room atmosphere, in contrast to 
working on a computer where the desk ergonomics and the screen luminance are adjustable.

To ensure a good optic comfort, four discomfort criteria are measured to give an assessment of the global comfort level (opposite of the discomfort level in fuzzy logic) and to feed into a control law in order to drive several actuators, such as automatic blinds and lights. Because the experimental conditions are real, glare cannot be considered only as a ratio between both ergorama and panorama zones but instead for every potential light source and its background. Thus the first criterion consists of detecting the high contrast zones in the field of view that could be considered as uncomfortable backlighting. Although the image processing only treats a picture from a given point of view according to the fisheye sensor location, potential positions can be identified where backlighting could occur; that means where it could be difficult to see the face of the person we are talking to (in case of facing a light source like a window). This image processing consists of identifying zones of high levels of luminance that could appear as uncomfortable sources if within the ergorama field or tiring sources if within the panorama field. These zones can be considered more or less visually aggressive according to the considered point of view. Backlighting and local glare detected in high contrast zones give the two first criteria of discomfort. These are respectively related to the number of uncomfortable zones, which can impair the quality of view of central subjects in the concerned scene, and the number of tiring zones, which alter only the global optic comfort.

The third criterion deals with the balance of light. Indeed a comfortable optic atmosphere in a room depends on the homogeneity of light that helps shadows and backlighting not to occur. Finally the fourth criterion is the light mean level required to correctly perform a task such as reading.

These four criteria have to be obtained from the fisheye video camera in order to feed an artificial optic comfort sensor. These measures are detailed in the next section.

However, the fisheye video camera may also provide control laws with some more formal measurements such as the state of blinds for instance (position), transmittance of windows (to balance the light coming through all the windows), the location of windows and lights, light distribution on the ceiling, etc.

\subsection{Implementation}

The global goal is to design smart devices that will control the optic and thermal comfort in a room by relying on natural and free solar energy. The optic sensor we refer to in this article is a part of the global system. To implement and develop either those devices or their algorithms, a room has been dedicated as an experimental room (Fig.1). This room is $5 \mathrm{~m}$ in width, $10 \mathrm{~m}$ in length, $2.5 \mathrm{~m}$ in height and includes 4 windows $(1.7 \times 1.3 \mathrm{~m})$ on a wall oriented at $25,7^{\circ}$ North-East. The building is in the city of Toulon in south of France and its GPS location is $43^{\circ} 07^{\prime}$ North and $5^{\circ} 56^{\prime}$ East. Even if the windowed façade concerned is North facing, the room is exposed to a high quantity of daylight because this region of
France is very sunny and also because several bright buildings and glazed facades cross the street reflect the sun light through the windows of our experimental room. The view from the $5^{\text {th }}$ floor is very open and wide and the landscape is diverse and composed of buildings (street environment), a small mountain $(600 \mathrm{~m}$ high, $3.3 \mathrm{~km}$ away from our institute) and sky.

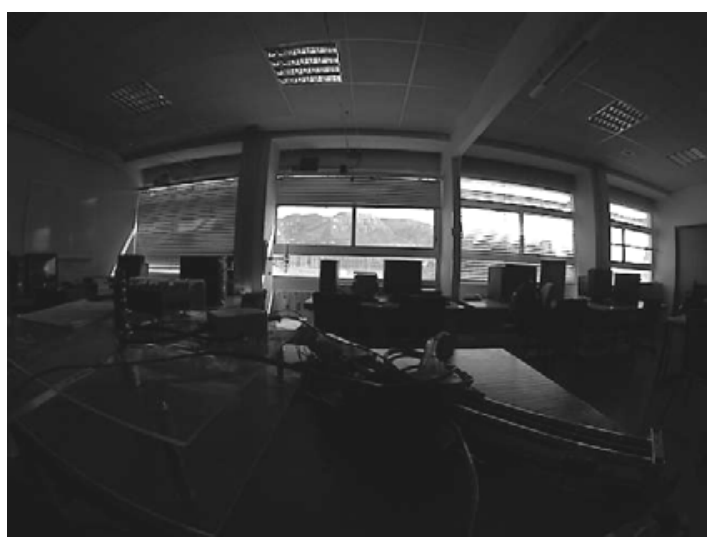

Fig. 1. Fisheye snap shot $\mathrm{I}_{\text {Global }}$

Two of the four windows of the experimental room are each equipped with individually driven blinds and a line of three halogen low voltage lights directed towards the ceiling which are hung from the window to the opposite wall. Thus half of the room can be automatically controlled whereas the second half is manually driven. The hardware and control laws for all the optic or thermal actuators are not detailed in this paper. LabVIEW 8.20 has been chosen to run the experimental room either for sensors or actuators.

The optic comfort sensor is based on a fisheye colour video camera AXIS 212 which has a 3 Mpixel resolution (2048×1536 pixels) and $140^{\circ}$ as aperture angle. The large pictures provided by the fisheye video camera allow simulation of the human field of view. The automatic white balance was turned off to guarantee good repeatability for any type of light. Snap shots are converted to grey scale pictures saved as 640x480 pixel jpeg files before being treated by different filters. These help us to analyse the light conditions in the room and interpret them as a human perceived optic comfort level. The first step is effected by series of classical image filters whereas the second part relies on fuzzy logic to provide a fuzzy sensor dedicated to visual comfort. Both parts will be detailed in following sections.

\subsection{Image processing}

To give the level of optic comfort, we will evaluate the influence of each discomfort parameter described in the section 3.1. Studied snap shots are supposed to simulate the field of view of a worker facing the windows (the most critical case) either standing or sat on a desk chair. The presented example (Fig. 1) shows a quite dark photo because of the backlighting and because the automatic white balance was deliberately turned off. The white balance was manually set to allow the video camera not to be dazzled by the high outdoor light (sky). The study of the discomfort level starts with a global picture of the room (Fig. 1) in which four 
windows in different positions are visible. Indeed from the left to the right, blinds are respectively shut for the whole window, shut only at the top, open for the whole window and folded up for the last one. The view of the mountain in the landscape illustrates easily the different states of the blinds.

The first two criteria to evaluate refer to uncomfortable and tiring zones as described in section 3.1.Our method to extract these zones is to detect high contrast zones and to compare their luminance to their background luminance. The first step consists of selecting the brightest zones in the scene by taking into account the mean luminance level of the whole picture. To avoid aberrations due to the noise or non-significant details in the image, we apply a quantification filter, which reduces the number of grey levels from 255 to 8 . This reduction is not performed as a linear regression but follows a logarithmic curve drawn to emphasize contrasts in darkest levels and generate another image, $\mathrm{I}_{\text {Post }}$.

This image is then passed through two other filters to extract the light sources and their surrounding contrast. A dilatation filter is applied to one image to widen the light sources and an erosion filter is applied to a duplicate image to stretch the light sources. These filters respectively give two new images $\mathrm{I}_{\text {Dil }}$ and $\mathrm{I}_{\text {Ero. }}$.

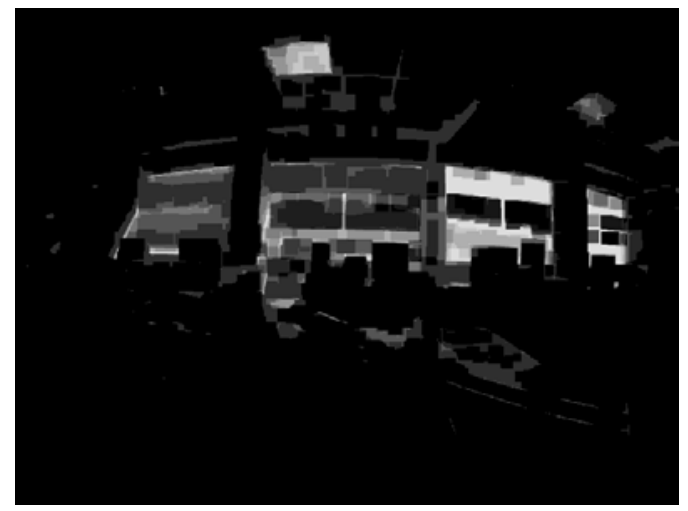

Fig. 2. $\mathrm{I}_{\text {Sub }}$ image

The subtraction of these two images, $\mathrm{I}_{\mathrm{Dil}}-\mathrm{I}_{\text {Ero }}$, gives a new image $\mathrm{I}_{\text {Sub }}$, which illustrates the luminance differences of the various light sources and their nearby background (Fig. 2).

In the next step, $\mathrm{I}_{\mathrm{Sub}}$ is passed through a differentiation filter in order to amplify the contrast and gives a new image $I_{\text {Diff. }}$. This filter changes the value of a pixel to give it the absolute value of its maximum deviation from its upper-left neighbours (1)(Fig. 3).

$P_{(i, j)}=\max \left\lfloor\left|P_{(i-1, j)}-P_{(i, j)}\right|,\left|P_{(i-1, j-1)}-P_{(i, j)}\right|,\left|P_{(i, j-1)}-P_{(i, j)}\right|\right\rfloor$

With the pixel arrangement as follows (Fig.3)

\begin{tabular}{|l|l|}
\hline$P_{i-1, j-1}$ & $P_{i, j-1}$ \\
\hline$P_{i-1, j}$ & $P_{i, j}$ \\
\hline
\end{tabular}

Fig. 3. Pixel arrangement for differentiation filter

Finally we will consider as a high contrast zone all the pixels of $\mathrm{I}_{\text {Diff }}$ that are 5 times higher than the mean luminance level of $\mathrm{I}_{\text {Post. }}$ Furthermore, a final test of the size of the zones will ensure that high contrast zones are composed of at least 25 pixels. This treatment, conducted using a bounding box processing method, allows us to focus on only significant zones bigger than the fovea zone of the human eye.

The high contrast zones will be classified as uncomfortable zones if within the ergorama or tiring zones if within the panorama. In the case of a wide dazzling zone, both criteria can contribute to a bad comfort level. Moreover, in this case, the light balance can be affected and thus it will also influence the comfort level.

The third criterion, the light balance, influences the optic comfort level because it can generate glare or involves both eyes being exposed to very different levels of light. This processing consists of computing the barycentre in the grey levels of an image. The global picture passes through a gamma correction filter (with $\gamma=2.5$ ) in order to emphasize contrast before undergoing light balance processing. The light balance level is then expressed as a Euclidian distance between the barycentre of the image and the optic centre. This distance is given as a number of pixels.

The fourth criterion chosen to assess the comfort level is the illumination level, which can be sufficient or not to comfortably perform a task of reading or chatting within the experimental room. This criterion is computed as the average of the grey level of all the pixels of the whole picture.

All these four criteria are computed in the same LabVIEW program on a common GUI (Fig. 4). The results of image processing are drawn as colour spots on the global snap shot to indicate the location of the barycentre, uncomfortable and tiring zones. Another part of the screen is dedicated to the fuzzy logic processing of our optic comfort sensor, which will be detailed in the next section.

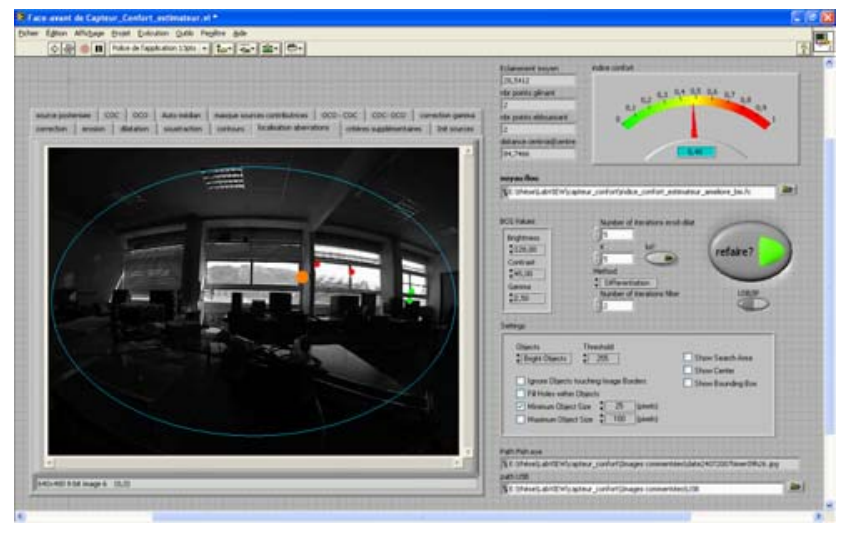

Fig. 4. Dashboard of image processing

\subsection{Fuzzy interpretation}

We try to model human optic comfort perception by using fuzzy logic and the four discomfort criteria presented above. We explain in this section how and why the fuzzy subsets were designed for each criterion. Even if a fuzzy system requires a learning process to be initialized, our fuzzy sensor should supply for most people a reliable rating related to optic 
atmosphere because it deals with discomfort criteria that are usually quite common to everyone.

So, the first input, the mean illumination, is represented by two fuzzy subsets, so that we take into account that a mean illumination higher than the threshold enables tasks such as reading or writing to be properly performed. With a low illumination level, those tasks cannot be comfortably and/or properly performed.

Table 1. Fuzzy subsets description

\begin{tabular}{|c|c|c|c|c|}
\hline \multicolumn{5}{|c|}{ Mean illumination (pixel level) } \\
\hline & $\begin{array}{c}\text { Left } \\
\text { bottom }\end{array}$ & Left top & $\begin{array}{l}\text { Right } \\
\text { top }\end{array}$ & $\begin{array}{l}\text { Right } \\
\text { bottom }\end{array}$ \\
\hline Low & 0 & 0 & 5 & 15 \\
\hline Medium & 5 & 15 & 20 & 20 \\
\hline \multicolumn{5}{|c|}{ Number of bothering zones } \\
\hline Small & 0 & 0 & 1,5 & 3 \\
\hline Medium & 1,5 & 3 & 3 & 4,5 \\
\hline High & 3 & 4,5 & 6 & 6 \\
\hline \multicolumn{5}{|c|}{ Number of tiring zones } \\
\hline & $\begin{array}{c}\text { Left } \\
\text { bottom }\end{array}$ & Left top & $\begin{array}{c}\text { Right } \\
\text { top }\end{array}$ & $\begin{array}{l}\text { Right } \\
\text { bottom }\end{array}$ \\
\hline Small & 0 & 0 & 0,75 & 1,5 \\
\hline Medium & 0,75 & 1,5 & 1,5 & 2,25 \\
\hline High & 1,5 & 2,5 & 3 & 3 \\
\hline \multicolumn{5}{|c|}{ Light balance(distance in pixels to the image centre) } \\
\hline Short & 0 & 0 & 75 & 150 \\
\hline Average & 75 & 150 & 150 & 225 \\
\hline Long & 150 & 225 & 300 & 300 \\
\hline \multicolumn{5}{|c|}{ Comfort level (output) } \\
\hline $\mathrm{Bad}$ & 0 & 0 & 0,2 & 0,4 \\
\hline Insufficient & 0,2 & 0,4 & 0,4 & 0,6 \\
\hline Correct & 0,4 & 0,6 & 0,6 & 0,8 \\
\hline Good & 0,6 & 0,8 & 0,8 & 1 \\
\hline
\end{tabular}

The second input (number of tiring zones) and the third one (number of uncomfortable zones) are drawn by three fuzzy subsets to give a smooth progression of zone detection and thus to improve the robustness of the algorithm. The last input, light balance, is split into three fuzzy subsets that follow the three zones of ergorama, panorama and monocular vision. These angular zones of view are converted to a distance from the optic centre of the considered picture. Experiments showed that a scene that is unbalanced in light creates discomfort independent of the location of the sources.

The output, visual comfort level, was divided into four fuzzy subsets in order to provide a higher output resolution and thus more nuances for the initialization phase of our fuzzy sensor. This way, we can more precisely describe the perception of luminous atmosphere degradations, which can appear progressively after eye adaptation. The output criterion fuzzy subsets relate to the comfort level during either reading or chatting tasks. All the fuzzy subsets are drawn as trapezes and detailed in table 1.

The fuzzy rules, presented just below, were defined in regard to basic laws of comfort described in the section 3.1 .

* A high number of uncomfortable zones implies a bad comfort level.
* A medium number of tiring zones implies a bad comfort level.

* A low mean illumination linearly decreases the output given from the same combination of the three other inputs with a medium mean illumination.

* The longer the light balance, the worse the comfort level.

We created a Sugeno type inference system with $\mathrm{min} / \mathrm{max}$ operators and a centroid defuzzification method. Our fuzzy sensor was simulated with Matlab R14 environment.

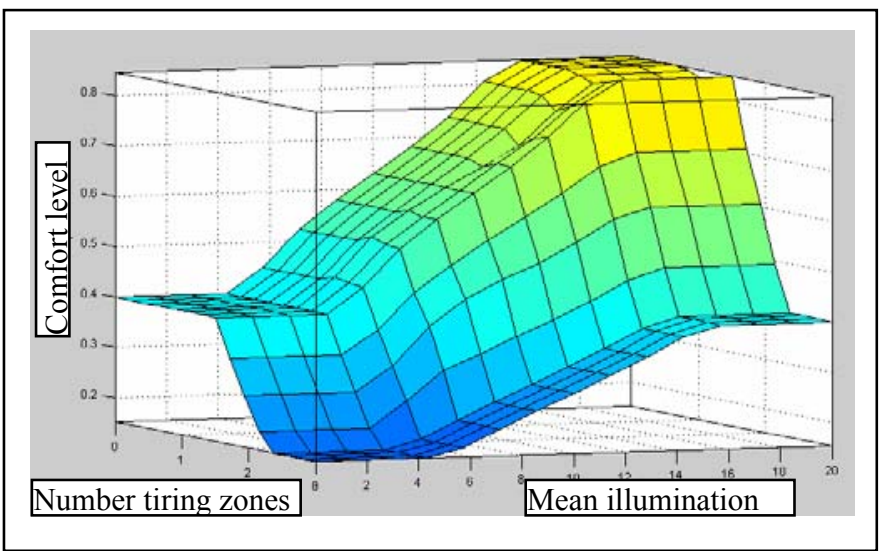

Fig. 5. Fuzzy optic comfort surface

Figure 5 shows the optic comfort level as a surface drawn as a function of both criteria, which are number of uncomfortable zones and the level of the mean illumination. The other criteria are equal to zero in this example. We notice the high influence of the number of tiring zones and the uncertainties related to their detection as explained before.

Above a threshold of two tiring zones, the comfort is very strongly affected, this describes glare occurrence between a light source and its environment. Even if the illumination level and the light balance are optimal and the number of tiring zones is null, the criterion related to glare limits the comfort level at 0,4 on a scale from 0 to 1 . That means that it is necessary to deal with glare level to insure a good comfort level.

However, the comfort level varies linearly with both the mean illumination level and the light balance and can be useful to influence the light atmosphere. When the trend is linear (and not close to the extremes of the range), the mean illumination level can slightly contribute towards an improved comfort level provided none of the other criteria change. The mean illumination depends on daylight through the windows but also on artificial lights, which can be easily controlled to follow a comfort trajectory or to adjust the comfort level.

\section{OPTIC SENSOR IN THE CONTROL LOOP}

The visual comfort sensor is primarily designed to work as a validation device; namely it assesses the comfort level in the room and then it is helpful to confirm the efficiency of different control laws for blinds or lights. Moreover this sensor can be introduced into the control loop to provide the controller with states related to many actuators and sensors 
(position of blinds, global illumination, light distribution...) (Fig. 6). This double function enables reliance on this sensor as a low-level feedback sensor but also as a high level sensor, which helps to choose the desired comfort trajectory to follow. The trajectory should respond to an optic atmosphere order without generating any discomfort configuration. This characteristic involves our system being robust in case of a quick change of outdoor light conditions (rapid appearance of a cloud, for instance).

To simplify the control of actuators, we have established cartographies of the comfort parameters concerned and the actuators states. It is easy to know that blinds will act on several criteria such as dazzling zones whereas the mean illumination will depend in part on the state of the lights.

There are several potential strategies to control the whole room. The first strategy is to focus only on the comfort index to maintain a constant level of light. In this case, the cartography of each actuator but also a global internal model will be used to draw a comfort path (the global optic model has not been presented in any papers yet). The second strategy is to apply a law based on the rejection of disturbances. In this case, a local solution will be generated, although this cannot ensure avoidance of uncomfortable configurations. The third strategy is to apply some main principles, which should contribute to generate comfortable configurations while properly controlling the light level (desired atmosphere). One of these principles is, for example, to balance the quantity of light coming through each window on the same façade. This method will reduce high contrast at least between neighbouring windows.

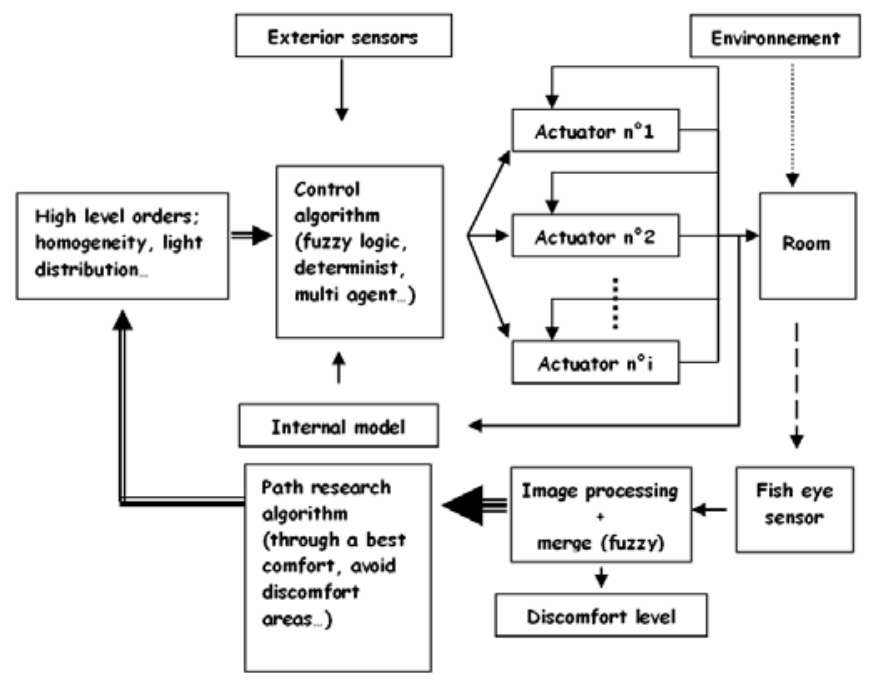

Fig. 6. Control architecture

The control laws expected to satisfy these different strategies are various. Indeed we anticipate to implement and compare the efficiency of a classical PID controller, multi-agent controller and fuzzy control laws. In addition to the comfort level, energy consumption will be measured to estimate the interest of these control laws.

\section{CONCLUSION}

A visual comfort sensor has been designed to give an objective and reliable measure related to the optic atmosphere perceived by people within a room. This artificial sensor based on a fuzzy logic kernel takes into account human perception and merges four discomfort criteria to provide an instant comfort assessment.

Moreover this fisheye video camera sensor supplies many interesting parameters that are very helpful to control the experimental room. This sensor completes favourably the rest of the room to assess its own performances and produce global comfort solutions.

Our forthcoming investigations will focus on the control of the whole room, which will rely on experimental measurements, internal physical models (actuators and states) and human perception models by using the visual sensor presented in this paper. This phase will tackle more precisely the aspects of energy consumption and thermal models.

\section{REFERENCES}

Barlow, S., Fiala, D. Occupant comfort in UK offices. How adaptive comfort theories might influence future low energy office refurbishment strategies. Energy \& Buildings (2007), doi:10.1016/j.enbuild.2007.02.002.

Jin Sook, L., Byoung Soo, K. Development of the nomograph for evalution on discomfort glare of windows. Solar Energy, 81, 2007, pp799-808.

Leclercq, M., Anthierens, C., Bideaux, E., Flambard, L. Régulation du confort visuel dans une pièce : analyse pour la mise en oeuvre. $2^{\text {ndes }}$ journées doctorales du Groupe de Recherche Modélisation, Analyse, Commande des systèmes Complexes, Reims, France, 9-11 juillet 2007, 6p.

Nazzal, A. A new evaluation mehod for daylight discomfort glare. International Journal of Industrial Ergonomics 35, 2005, pp295-306.

Serra, R. Chapter 6 - Daylighting. Renewable and Sustainable Energy Reviews. 1998, pp 115-155.

Vischer, J.C. Space meets status: Designing workspace performance. Routledge, 184 p, London Spon Press, 2005.

Werner, K.E., Osterhaus. Discomfort glare assessment and prevention for daylight applications in office environments. Solar Energy 79, 2005, pp140-158.

Wienold, J., Christoffersen, J. Evaluation methods and development of a new glare prediction model for daylight environments with the use of CCD cameras. Energy and buildings 38, 2006, pp743-757.

Wonwoo, K., Yasuko, K. Glare constant Gw for the evaluation of discomfort from windows. Solar Energy 78, 2005, pp 105-111. 